\title{
AN INDEX FORMULA FOR THE DEGREE OF (S)+-MAPPINGS ASSOCIATED WITH ONE-DIMENSIONAL $p$-LAPLACIAN
}

JUN KOBAYASHI AND MITSUHARU ÔTANI

Received 11 August 2003

The topological degree for $(S)_{+}$-mappings concerning a nonlinear eigenvalue problem associated with one-dimensional $p$-Laplacian is evaluated. The result is applied to a variational inequality, where the multiple existence of solutions is discussed.

\section{Introduction}

In this paper, we evaluate the topological degree for $(S)_{+}$-mappings concerning the following nonlinear eigenvalue problem:

$$
\begin{gathered}
-\left(\left|u^{\prime}(x)\right|^{p-2} u^{\prime}(x)\right)^{\prime}=\mu|u(x)|^{p-2} u(x), \quad x \in(0,1), \\
u(0)=u(1)=0,
\end{gathered}
$$

where $p>1$ and $\mu \in \mathbb{R}$. It is shown by the second author [12] that all eigenvalues for (1.1) are explicitly written in terms of the beta function as follows:

$$
\mu_{k}(p)=(p-1)\left\{k \frac{2}{p} B\left(\frac{1}{p}, 1-\frac{1}{p}\right)\right\}^{p}
$$

and that $\mu_{k}(p)$ are all simple.

Define the $(\mathrm{S})_{+}$-mapping (see [2]) $T_{\mu}^{p}: W_{0}^{1, p}(0,1) \rightarrow W^{-1, p^{\prime}}(0,1)$ by

$$
\left\langle T_{\mu}^{p}(u), v\right\rangle=\int_{0}^{1}\left|u^{\prime}\right|^{p-2} u^{\prime} v^{\prime} d x-\mu \int_{0}^{1}|u|^{p-2} u v d x, \quad u, v \in W_{0}^{1, p}(0,1) .
$$

Then, we have the following theorem.

Theorem 1.1. Let $\mu \in\left(\mu_{k}(p), \mu_{k+1}(p)\right)$ for some $k \in \mathbb{N} \cup\{0\}$. Then,

$$
\operatorname{deg}_{(\mathrm{S})_{+}}\left(T_{\mu}^{p}, U(0, \rho), 0\right)=(-1)^{k} \quad \forall \rho>0,
$$

where $U(0, \rho)$ is the open ball in $W_{0}^{1, p}(0,1)$ centered at the origin with radius $\rho$. (As for $k=0$, it is understood that $\mu_{0}=-\infty$.) 
Concerning the Leray-Schauder degree, the corresponding result is obtained in [3]. As for the higher-dimensional case, that is, the case where the interval $(0,1)$ is replaced by a bounded domain $\Omega$ in $\mathbb{R}^{N}$ (and $\mu<\mu_{2}(p)$ ), see [4] (Leray-Schauder degree) and [5] (degree for $(\mathrm{S})_{+}$-mappings).

These kind of results are useful for the study of the (multiple) existence of the equations of the type

$$
\begin{gathered}
-\operatorname{div}\left(|\nabla u|^{p-2} \nabla u\right)=f(x, u) \quad \text { in } \Omega, \\
u=0 \quad \text { on } \partial \Omega
\end{gathered}
$$

as well as the variational inequalities of the type

$$
\int_{\Omega}|\nabla u|^{p-2} \nabla u \cdot \nabla(v-u) d x+\varphi(v)-\varphi(u) \geq \int_{\Omega} f(x, u)(v-u) d x \quad \forall v \in W_{0}^{1, p}(\Omega),
$$

where $f$ is a Carathéodory function and $\varphi$ is a lower semicontinuous convex function.

The index formulas for mappings of class $(S)_{+}$or "densely defined" mappings which satisfy a variant of $(S)_{+}$condition have been investigated in $[7,8,9,13]$ in abstract settings. However, they assume that the leading term of mappings does not degenerate in a sense, and hence their results cannot be applied directly to our problem.

To prove Theorem 1.1, we employ a technique similar to those in $[3,4]$, a homotopic deformation along $p$ to the case $p=2$. In [3], such a deformation is applied in $C[0,1]$, the same Banach space where the degree is considered in the formula corresponding to (1.4). On the other hand, in [4], the corresponding result to Theorem 1.1 is considered in $W_{0}^{1, p}(\Omega)$, which varies with $p$, so more delicate arguments are required. One needs a lemma which provides a connection between two degrees in different Banach spaces, the degree in $W_{0}^{1, p}(\Omega)$, and the degree in $L^{q}(\Omega)$ for some fixed $q$ (see [4, Lemma 2.4]). Then, a homotopic deformation is used in $L^{q}(\Omega)$.

Our strategy is similar to that of the latter case. We employ the degree theory for subdifferential operators which is developed in our previous work [10]. It is shown that the left-hand side of (1.4) coincides with the degree in $L^{2}(0,1)$ for some mapping given as the sum of a subdifferential operator and a perturbation associated with (1.1). Then, a homotopic deformation along $p$ is applied in the fixed space $L^{2}(0,1)$.

This paper is composed of four sections. In Section 2, we recall some basic facts on the degree for $(S)_{+}$-mappings as well as the degree for subdifferential operators. A proof of Theorem 1.1 is given in Section 3, where the procedure mentioned above is carried out. In Section 4, we give an example of applications to variational inequalities, where the multiple existence of solutions is discussed.

\section{Preliminaries}

If $X$ is a Banach space, then the norm of $X$ will be denoted by $\|\cdot\|_{X}$ or $|\cdot|_{X}$. We denote by $\langle\cdot, \cdot\rangle_{X}$ the duality pairing between $X$ and its dual $X^{*}$ and by $U_{X}(u, r)$ the open ball of $X$ centered at $u$ with radius $r>0$. For a subset $A$ of $X$, the closure and the boundary of $A$ with respect to the topology of $X$ are designated by $\bar{A}^{X}$ and $\partial_{X} A$, respectively. 
Usually, $\operatorname{deg}(M, D, f)$ stands for the degree for a mapping $M$ relative to a bounded open subset $D$ at a point $f$. By substituting $M-f$ for $M$, we can always assume that $f=0$. We simply denote $\operatorname{deg}(M, D)$ instead of $\operatorname{deg}(M, D, 0)$.

In this paper, we treat three types of degrees, the Brouwer degree, the degree for $(S)_{+}-$ mappings (with maximal monotone perturbation), and the degree for subdifferential operators in Hilbert spaces developed in [10]. They will be denoted by $d(\cdot, \cdot), \operatorname{deg}_{(S)_{+}}(\cdot, \cdot)$, and $\operatorname{deg}_{H}(\cdot, \cdot)$, respectively.

2.1. Degree for $(S)_{+}$-mappings. Let $X$ be a real reflexive Banach space. A single-valued mapping $S$ from $D(S) \subset X$ into $X^{*}$ is said to be class $(S)_{+}$if for any sequence $\left(u_{n}\right)$ of $D(S)$, the conditions

$$
u_{n} \rightarrow u \text { weakly in } X, \quad \limsup _{n \rightarrow \infty}\left\langle S\left(u_{n}\right), u_{n}-u\right\rangle_{X} \leq 0
$$

imply that $u_{n} \rightarrow u$ strongly.

We here recall how to define the degree for $S$.

Let $D$ be a bounded open subset of $X$ and let $S$ be a demicontinuous $(S)_{+}$-mapping from $\bar{D}$ into $X^{*}$. Let $\left\{X_{\alpha}: \alpha \in A\right\}$ be the family of all finite-dimensional subspaces of $X$ and let $S_{\alpha}$ be the Galerkin approximation of $S$ with respect to $X_{\alpha}$, that is,

$$
\left\langle S_{\alpha}(u), v\right\rangle_{X_{\alpha}}=\langle S(u), v\rangle_{X} \quad \forall u \in \bar{D} \cap X_{\alpha}, \forall v \in X_{\alpha} .
$$

Then, $\operatorname{deg}_{(S)_{+}}(S, D)$ is defined by

$$
\operatorname{deg}_{(S)_{+}}(S, D):=d\left(S_{\alpha}, D \cap X_{\alpha}\right)
$$

for $X_{\alpha}$ large enough (in the sense of inclusion). Moreover, as for the case where $X$ is separable and $S$ is bounded, we can easily see that the role of $\left\{X_{\alpha}\right\}$ can be played by a countable subfamily $\left\{X_{i}: i \in \mathbb{N}\right\} \subset\left\{X_{\alpha}\right\}$ which satisfy

$$
\bar{\bigcup}_{i \in \mathbb{N}} X_{i}^{X}=X
$$

(see the proof of [2, Theorem 4]).

2.2. Degree for subdifferential operators. Let $H$ be a real separable Hilbert space with the inner product $(\cdot, \cdot)_{H}$.

Definition 2.1. Denote by $\Phi_{C}(H)$ the collection of lower semicontinuous convex functions $\varphi$ from $H$ into $[0,+\infty]$ which satisfy the following conditions:

(A.0) $\varphi(0)=0$,

(A.C) for each $L \in(0,+\infty)$, the level set $\left\{u \in H: \varphi(u)+|u|_{H}^{2} \leq L\right\}$ is compact in $H$.

For $\varphi \in \Phi_{C}(H)$ and $u \in D(\varphi)(=\{u \in H: \varphi(u)<+\infty\})$, define the subdifferential $\partial \varphi(u)$ of $\varphi$ at $u$ by

$$
\partial \varphi(u)=\left\{v \in H: \varphi(w)-\varphi(u) \geq(v, w-u)_{H} \forall w \in H\right\} .
$$


Then, as it is well known, $\partial \varphi$ becomes a maximal monotone operator in $H$. For $\lambda>0$, we denote by $J_{\lambda}$ and $\partial \varphi_{\lambda}$ the resolvent and the Yosida approximation of $\varphi$, respectively, that is,

$$
J_{\lambda}=(I+\lambda \partial \varphi)^{-1}, \quad \partial \varphi_{\lambda}=\frac{1}{\lambda}\left(I-J_{\lambda}\right)
$$

where $I$ is the identity on $H$. It follows from (A.0) that $0 \in \partial \varphi(0)$ and hence $J_{\lambda} 0=0$, $\partial \varphi_{\lambda}(0)=0,\left|J_{\lambda} u\right|_{H} \leq|u|_{H}$, and $\left|\partial \varphi_{\lambda}(u)\right|_{H} \leq|u|_{H} / \lambda$ for all $u \in H$ and $\lambda>0$.

We define a class of homotopy in $\Phi_{C}(H)$ likewise.

Definition 2.2. Denote by $\Phi_{C}^{\text {hom }}(H)$ the collection of families $\left\{\varphi^{t}: t \in[0,1]\right\}$ of members of $\Phi_{C}(H)$ which satisfy the following conditions:

$(\text { A.C })^{h}$ for each $L \in(0,+\infty)$, the set

$$
\bigcup_{t \in[0,1]}\left\{u \in H: \varphi^{t}(u)+|u|_{H}^{2} \leq L\right\}
$$

is precompact in $H$,

$(\mathrm{A} . \mathrm{P})^{h}\left\{\partial \varphi^{t}: t \in[0,1]\right\}$ is a pseudomonotone homotopy of maximal monotone operators, that is, if $t_{n} \rightarrow t$ in $[0,1]$ and $[u, v] \in \partial \varphi^{t}$, then there exist $\left[u_{n}, v_{n}\right] \in \partial \varphi^{t_{n}}$ such that $u_{n} \rightarrow u$ and $v_{n} \rightarrow v$ strongly.

(Concerning other type of conditions equivalent to (A.P $)^{h}$ see [2, Definition 8] or [11, Definition 2.2].)

In [10], we introduced two classes of multivalued perturbations (and their homotopies). For our purpose here, we only recall one of them in a restricted form (especially we consider a class of single-valued perturbations).

Definition 2.3. For a given $\varphi \in \Phi_{C}(H)$, denote by $\mathscr{B}_{1}(\varphi)$ the collection of mappings $B$ from $D(B) \subset H$ into $H$ which satisfy the following conditions:

(A.1) $D(B) \supset D(\partial \varphi)$,

(A.2) if $\left[u_{n}, v_{n}\right] \in \partial \varphi, u_{n} \rightarrow u$ strongly, $v_{n} \rightarrow v$ weakly and $B u_{n} \rightarrow b$ weakly, then $b=$ $\mathrm{Bu}$,

(A.3) there exist $k \in(0,1), \alpha \in(0,2)$, and a positive, monotone increasing function $l$ such that

$$
|B u|_{H}^{2} \leq k|v|_{H}^{2}+l\left(|u|_{H}\right)\left\{(\varphi(u))^{\alpha}+1\right\} \quad \forall[u, v] \in \partial \varphi .
$$

Definition 2.4. For a given $\left\{\varphi^{t}: t \in[0,1]\right\} \in \Phi_{C}^{\text {hom }}(H)$, denote by $\mathscr{B}_{1}^{\text {hom }}\left(\left\{\varphi^{t}\right\}\right)$ the collection of families $\left\{B^{t}: t \in[0,1]\right\}$ of mappings in $\mathscr{B}_{1}$ which satisfy the following conditions:

$(\mathrm{A} .2)^{h}$ if $t_{n} \rightarrow t,\left[u_{n}, v_{n}\right] \in \partial \varphi^{t_{n}}, u_{n} \rightarrow u$ strongly, $v_{n} \rightarrow v$ weakly, and $B^{t_{n}} u_{n} \rightarrow b$ weakly, then $b=B^{t} u$,

$(\text { A.3 })^{h}$ condition (A.3) is satisfied uniformly in $t$, that is, the constants $k, \alpha$ and the function $l$ can be chosen independently of $t$. 
Let $\varphi \in \Phi_{C}(H)$ and $B \in \mathscr{B}_{1}(\varphi)$. We here sketch how to define the degree for $\partial \varphi+B$.

Since $H$ is separable, there exists a sequence $\left(H_{i}\right)_{i \in \mathbb{N}}$ of finite-dimensional subspaces such that

$$
H_{1} \subset H_{2} \subset \cdots \subset H_{i} \subset \cdots, \quad \widehat{\bigcup}_{i \in \mathbb{N}} H_{i}{ }^{H}=H .
$$

For $i \in \mathbb{N}$ and $\lambda>0$, we put $B_{i, \lambda}:=P_{i} \circ B \circ J_{\lambda}$, where $P_{i}$ is the orthogonal projection from $H$ onto $H_{i}$. It easily follows from (A.C) that $J_{\lambda}$ is a compact mapping and so is $B_{i, \lambda}$ by (A.2) and (A.3). Hence, $\partial \varphi_{\lambda}+B_{i, \lambda}$ becomes a continuous mapping of class $(\mathrm{S})_{+}$(we identify $H^{*}$ with $H)$. Suppose now $0 \notin(\partial \varphi+B)\left(\partial_{H} D\right)$, where $D$ is a bounded open subset of $H$. Then, $\operatorname{deg}_{H}(\partial \varphi+B, D)$ is defined by

$$
\operatorname{deg}_{H}(\partial \varphi+B, D):=\operatorname{deg}_{(S)_{+}}\left(\partial \varphi_{\lambda}+B_{i, \lambda}, D\right)
$$

for sufficiently small $\lambda>0$ and for sufficiently large $i \in \mathbb{N}$.

In the following, for a mapping $M$ on $H$, we denote by $M_{i}$ the Galerkin approximation of $M$ with respect to $H_{i}$ :

$$
\left\langle M_{i}(u), v\right\rangle_{H_{i}}=(M(u), v)_{H} \quad \forall u \in D(M) \cap H_{i}, \forall v \in H_{i} .
$$

It then holds that for all $u \in H_{i}$,

$$
M_{i}(u)=0 \text { in }\left(H_{i}\right)^{*} \Longleftrightarrow P_{i} M(u)=0 \text { in } H .
$$

We also note that if $i \geq j$, then $\left(P_{j} \circ M\right)_{i}$ coincides with $M_{i}$. Therefore, by (2.3) (and the fact mentioned below it) and by (2.10), we have

$$
\operatorname{deg}_{H}(\partial \varphi+B, D)=d\left(\left(\partial \varphi_{\lambda}+B \circ J_{\lambda}\right)_{i}, D \cap H_{i}\right)
$$

for sufficiently small $\lambda>0$ and for sufficiently large $i \in \mathbb{N}$.

Let $H=L^{2}(0,1)$. For $p \in(1, \infty)$, define $\varphi^{p}: H \rightarrow[0,+\infty]$ by

$$
\varphi^{p}(u):= \begin{cases}\int_{0}^{1}\left|u^{\prime}(x)\right|^{p} d x & \text { if } u \in W_{0}^{1, p}(0,1) \\ +\infty & \text { otherwise. }\end{cases}
$$

Then, it easily follows that $\varphi^{p} \in \Phi_{C}(H)$. Moreover, $\partial \varphi^{p}(u)=-\left(\left|u^{\prime}\right|{ }^{p-2} u^{\prime}\right)^{\prime}$ in the sense of distribution with the domain

$$
D\left(\partial \varphi^{p}\right)=\left\{u \in W_{0}^{1, p}(0,1):\left(\left|u^{\prime}\right|^{p-2} u^{\prime}\right)^{\prime} \in H\right\} .
$$

For $p \in(1, \infty)$ and $\mu \in \mathbb{R}$, define $B_{\mu}^{p}: L^{2(p-1)}(0,1) \cap H \subset H \rightarrow H$ by

$$
B_{\mu}^{p} u:=-\mu|u|^{p-2} u
$$

Then, $B_{\mu}^{p} \in \mathscr{B}_{1}\left(\varphi^{p}\right)$ holds. Indeed, the verification of (A.1) is clear. The condition (A.2) follows from the fact that $B_{\mu}^{p}$ is weakly-strongly continuous from $W_{0}^{1, p}(0,1)$ into $H$. 
As for the boundedness condition (A.3), by the Gagliardo-Nirenberg inequality, there exist $C_{1}=C_{1}(p)>0, \alpha \in[0,2 / 3)$, and $\beta \in(0,2(p-1)]$ such that

$$
\left|B_{\mu}^{p} u\right|_{H}^{2} \leq \mu^{2} C_{1}\left\{\left(\varphi^{p}(u)\right)^{\alpha}|u|_{H}^{\beta}\right\} \quad \forall u \in W_{0}^{1, p}(0,1) .
$$

Hence, (A.3) follows.

\section{Proof of Theorem 1.1}

In this section, let $V_{p}$ and $H$ denote $W_{0}^{1, p}(0,1)$ and $L^{2}(0,1)$, respectively. Then,

$$
V_{p} \subset H \equiv H^{*} \subset V_{p}^{*},
$$

where each injection is dense and compact. Let $T_{\mu}^{p}, \varphi^{p}$, and $B_{\mu}^{p}$ be given in (1.3), (2.14), and (2.16), respectively. It then follows from (2.15) and (2.17) that, for all $u \in V_{p}$,

$$
\begin{gathered}
T_{\mu}^{p}(u) \in H \Longleftrightarrow u \in D\left(\partial \varphi^{p}\right), \\
u \in D\left(\partial \varphi^{p}\right) \Longrightarrow T_{\mu}^{p}(u)=\partial \varphi^{p}(u)+B_{\mu}^{p}(u) .
\end{gathered}
$$

Moreover, there exists a sequence $\left(H_{i}\right)_{i \in \mathbb{N}}$ of finite-dimensional subspaces which satisfies (2.9) and

$$
H_{i} \subset D\left(\partial \varphi^{p}\right) \subset V_{p}=D\left(\varphi^{p}\right) \subset H \quad \forall i \in \mathbb{N}, \forall p \in(1,+\infty)
$$

and

$$
\bar{\bigcup}_{i \in \mathbb{N}} H_{i}{ }^{P}=V_{p} .
$$

We also notice that for each $L>0$, the level set $\left\{u \in H: \varphi^{p}(u)+|u|_{H}^{2} \leq L\right\}$ is bounded in $V_{p}$.

Now, let $p \in(1, \infty)$ and $\mu \neq \mu_{k}(p)$ for all $k \in \mathbb{N}$. We are going to show

$$
\operatorname{deg}_{H}\left(\partial \varphi^{p}+B_{\mu}^{p}, U_{H}(0, r)\right)=\operatorname{deg}_{(\mathrm{S})_{+}}\left(T_{\mu}^{p}, U_{V_{p}}(0, \rho)\right) \quad \forall r, \rho>0
$$

by using the homotopy between the Galerkin approximations

$$
(1-t)\left(\partial \varphi_{\lambda}^{p}+B_{\mu}^{p} \circ J_{\lambda}\right)_{i}+t\left(T_{p}^{\mu}\right)_{i}
$$

In the following three lemmas, we drop $p$ and $\mu$ for simplicity.

LEMMA 3.1. Let $r_{1}>0$ and $r_{2}>0$ be such that $U_{H}\left(0, r_{1}\right) \supset{\overline{U_{V}\left(0, r_{2}\right)}}^{V}$. Then, there exists $i_{0} \in \mathbb{N}$ such that for all $i \geq i_{0}$,

$$
T_{i}(u) \neq 0 \quad \forall u \in H_{i} \cap\left(U_{H}\left(0, r_{1}\right) \backslash{\overline{U_{V}\left(0, r_{2}\right)}}^{V}\right) .
$$


Proof. Suppose that the assertion of the lemma was false. Then, we could find sequences $\left(i_{n}\right)$ and $\left(u_{n}\right)$ such that $i_{n} \rightarrow \infty, u_{n} \in H_{i_{n}},\left|u_{n}\right|_{H}<r_{1},\left\|u_{n}\right\|_{V} \geq r_{2}$, and $T_{i_{n}}\left(u_{n}\right)=0$. Especially, we have

$$
0=\left\langle T_{i_{n}}\left(u_{n}\right), u_{n}\right\rangle_{H_{i}}=\left(\partial \varphi\left(u_{n}\right)+B\left(u_{n}\right), u_{n}\right)_{H},
$$

which implies

$$
p \varphi\left(u_{n}\right)=\left(\partial \varphi\left(u_{n}\right), u_{n}\right)_{H}=-\left(B\left(u_{n}\right), u_{n}\right)_{H} \leq \frac{1}{2}\left|B\left(u_{n}\right)\right|_{H}^{2}+\frac{1}{2}\left|u_{n}\right|_{H}^{2}
$$

Therefore, it follows from (2.17) that $\varphi\left(u_{n}\right)$ is bounded as $n \rightarrow \infty$. Hence, $\left\|u_{n}\right\|_{V}$ is bounded, and so is $\left\|T\left(u_{n}\right)\right\|_{V^{*}}$. Passing to subsequence if necessary, we may assume that $u_{n} \rightarrow u$ weakly in $V$. Then, taking a sequence $\left(v_{n}\right)$ such that $v_{n} \in H_{n}$ and $v_{n} \rightarrow u$ strongly in $V$ (see (3.4)), we get

$$
\lim _{n \rightarrow \infty}\left\langle T\left(u_{n}\right), u_{n}-u\right\rangle_{V}=\lim _{n \rightarrow \infty}\left\langle T_{i_{n}}\left(u_{n}\right), u_{n}-v_{n}\right\rangle_{H_{i}}+\lim _{n \rightarrow \infty}\left\langle T\left(u_{n}\right), v_{n}-u\right\rangle_{V}=0 .
$$

Since $T$ is of class $(\mathrm{S})_{+}$, it follows that $u_{n} \rightarrow u$ strongly in $V$ (and hence $\|u\|_{V} \geq r_{2}$ ). From the continuity of $T$, we easily deduce $T(u)=0$, which implies $u=0$. Thus, we are led to a contradiction.

Let $r, \rho>0$. By choosing $r_{2}>0$ such that $U_{H}(0, r) \supset{\overline{U_{V}\left(0, r_{2}\right)}}^{V}$ and applying the lemma above and the excision property of degree, we deduce

$$
\begin{aligned}
\operatorname{deg}_{(S)_{+}}\left(T, U_{V}(0, \rho)\right) & =\operatorname{deg}_{(S)_{+}}\left(T, U_{V}\left(0, r_{2}\right)\right) \\
& =d\left(T_{i}, U_{V}\left(0, r_{2}\right) \cap H_{i}\right) \\
& =d\left(T_{i}, U_{H}(0, r) \cap H_{i}\right)
\end{aligned}
$$

for large $i \in \mathbb{N}$.

Lemma 3.2. Let $r>0$. Then, there exists $\lambda_{0}>0$ such that for all $\lambda \in\left(0, \lambda_{0}\right)$,

$$
(1-t)\left(\partial \varphi_{\lambda}(u)+B\left(J_{\lambda} u\right)\right)+t T(u) \neq 0 \quad \forall u \in V \cap \partial_{H} U_{H}(0, r), \forall t \in[0,1]
$$

Proof. Assuming the contrary, we could find sequences $\left(\lambda_{n}\right),\left(u_{n}\right)$, and $\left(t_{n}\right)$ such that $\left|u_{n}\right|_{H}=r, \lambda_{n} \rightarrow 0, t_{n} \rightarrow t$ in $[0,1]$ and

$$
\left(1-t_{n}\right)\left\{\partial \varphi_{\lambda_{n}}\left(u_{n}\right)+B\left(J_{\lambda_{n}} u_{n}\right)\right\}+t_{n} T\left(u_{n}\right)=0
$$

We have $t_{n} \neq 0$ since $\partial \varphi\left(J_{\lambda} u\right)+B\left(J_{\lambda} u\right)=0$ and $\mu \neq \mu_{k}(p)$ imply $u=0$. Therefore, $T\left(u_{n}\right) \in$ $H$, and hence, by (3.2),

$$
\left(1-t_{n}\right)\left\{\partial \varphi_{\lambda_{n}}\left(u_{n}\right)+B\left(J_{\lambda_{n}} u_{n}\right)\right\}+t_{n}\left\{\partial \varphi\left(u_{n}\right)+B\left(u_{n}\right)\right\}=0
$$


Multiplying this by $u_{n}$ yields

$$
\begin{aligned}
\left(1-t_{n}\right) & \varphi\left(J_{\lambda_{n}} u_{n}\right)+t_{n} \varphi\left(u_{n}\right) \\
\leq & \left(1-t_{n}\right)\left(\partial \varphi_{\lambda_{n}}\left(u_{n}\right), u_{n}\right)_{H}+t_{n}\left(\partial \varphi\left(u_{n}\right), u_{n}\right)_{H} \\
= & -\left(1-t_{n}\right)\left(B\left(J_{\lambda_{n}} u_{n}\right), u_{n}\right)_{H}-t_{n}\left(B\left(u_{n}\right), u_{n}\right)_{H} \\
\leq & \frac{1}{2}\left(1-t_{n}\right)\left|B\left(J_{\lambda_{n}} u_{n}\right)\right|_{H}^{2}+\frac{1}{2} t_{n}\left|B\left(u_{n}\right)\right|_{H}^{2}+\left|u_{n}\right|_{H}^{2} .
\end{aligned}
$$

By (2.17), this inequality implies that $\left(1-t_{n}\right) \varphi\left(J_{\lambda_{n}} u_{n}\right)$ and $t_{n} \varphi\left(u_{n}\right)$ are bounded as $n \rightarrow \infty$. Since $\varphi\left(J_{\lambda_{n}} u_{n}\right) \leq \varphi\left(u_{n}\right)$, it follows that $\varphi\left(J_{\lambda_{n}} u_{n}\right)$ is also bounded. By (2.17) again, $\left|B\left(J_{\lambda_{n}} u_{n}\right)\right|_{H}^{2}$ and $t_{n}\left|B\left(u_{n}\right)\right|_{H}^{2}$ are bounded. Here, since

$$
\left(\partial \varphi_{\lambda_{n}}\left(u_{n}\right), \partial \varphi\left(u_{n}\right)\right)_{H} \geq\left|\partial \varphi_{\lambda_{n}}\left(u_{n}\right)\right|_{H}^{2} \geq 0
$$

(cf. Brézis [1, Proposition 2.6]), multiplying (3.14) by $\partial \varphi_{\lambda_{n}}\left(u_{n}\right)$, we easily see that $\left|\partial \varphi_{\lambda_{n}}\left(u_{n}\right)\right|_{H}^{2}$ is bounded. Hence, by (3.14), the boundedness of $t_{n}\left|\partial \varphi\left(u_{n}\right)\right|_{H}$ is also derived. Accordingly, we may assume that

$$
\begin{array}{ll}
J_{\lambda_{n}} u_{n} \longrightarrow u & \text { strongly in } H, \\
\partial \varphi_{\lambda_{n}}\left(u_{n}\right)=\partial \varphi\left(J_{\lambda_{n}} u_{n}\right) \rightarrow v & \text { weakly in } H, \\
B\left(J_{\lambda_{n}} u_{n}\right) \rightarrow b & \text { weakly in } H
\end{array}
$$

for some $u, v, b \in H$. Then we have $v=\partial \varphi(u)$ and $b=B(u)$ by the demiclosedness of $\partial \varphi$ and by (A.2), respectively. Moreover, since $\left|u_{n}-J_{\lambda_{n}} u_{n}\right|_{H}=\lambda_{n}\left|\partial \varphi_{\lambda_{n}}\left(u_{n}\right)\right|_{H}$, we deduce that

$$
u_{n} \longrightarrow u \quad \text { strongly in } H
$$

(and hence $|u|_{H}=r$ ).

If $t \neq 0$, then $\left|\partial \varphi\left(u_{n}\right)\right|_{H}$ and $\left|B\left(u_{n}\right)\right|_{H}$ are bounded. Therefore, by the same argument as above, we conclude that (for some subsequence)

$$
\partial \varphi\left(u_{n}\right) \rightarrow \partial \varphi(u), \quad B\left(u_{n}\right) \rightarrow B(u) \quad \text { weakly in } H .
$$

Letting $n \rightarrow \infty$ in (3.14), we get $\partial \varphi(u)+B(u)=0$, which implies $u=0$. This contradicts $|u|_{H}=r$.

Suppose now $t=0$, that is, $t_{n} \rightarrow 0$. Since $t_{n} \varphi\left(u_{n}\right)=\varphi\left(t_{n}^{1 / p} u_{n}\right)$ is bounded, so is $t_{n}^{1 / p}\left\|u_{n}\right\|_{V}$. Therefore, $t_{n}^{1 /(p-1)} u_{n} \rightarrow 0$ strongly in $V$, and hence

$$
t_{n} T\left(u_{n}\right)=T\left(t_{n}^{1 /(p-1)} u_{n}\right) \longrightarrow 0 \quad \text { strongly in } V^{*}
$$

Thus, similar to the case $t \neq 0$, letting $n \rightarrow \infty$ in (3.13) leads to a contradiction.

Lemma 3.3. Let $r>0$, and let $\lambda$ be fixed to satisfy (3.12). Then, there exists $i_{0}=i_{0}(\lambda) \in \mathbb{N}$ such that for all $i \geq i_{0}$

$$
(1-t)\left(\partial \varphi_{\lambda}(u)+B\left(J_{\lambda} u\right)\right)_{i}+t T_{i}(u) \neq 0 \quad \forall u \in H_{i} \cap \partial_{H} U_{H}(0, r), \forall t \in[0,1] .
$$


Proof. If not, there exist sequences $\left(i_{n}\right),\left(u_{n}\right)$, and $\left(t_{n}\right)$ such that $i_{n} \rightarrow \infty, u_{n} \in H_{i_{n}},\left|u_{n}\right|_{H}=$ $r, t_{n} \rightarrow t$ in $[0,1]$ and

$$
\left(1-t_{n}\right)\left(\partial \varphi_{\lambda}\left(u_{n}\right)+B\left(J_{\lambda_{n}} u_{n}\right)\right)_{i}+t_{n} T_{i_{n}}\left(u_{n}\right)=0
$$

Since $u_{n} \in H_{i_{n}} \subset D(\partial \varphi)$, we have $T\left(u_{n}\right) \in H$. Therefore, by (2.12),

$$
\left(1-t_{n}\right) P_{i_{n}}\left\{\partial \varphi_{\lambda}\left(u_{n}\right)+B\left(J_{\lambda} u_{n}\right)\right\}+t_{n} P_{i_{n}}\left\{\partial \varphi\left(u_{n}\right)+B\left(u_{n}\right)\right\}=0
$$

Since $\left|u_{n}\right|_{H}=r$ and $\left|\partial \varphi_{\lambda}\left(u_{n}\right)\right|_{H} \leq\left|u_{n}\right|_{H} / \lambda$, we may assume that

$$
\begin{gathered}
u_{n} \rightarrow u \quad \text { weakly in } H, \\
\partial \varphi_{\lambda}\left(u_{n}\right)=\partial \varphi\left(J_{\lambda} u_{n}\right) \rightarrow v^{*} \quad \text { weakly in } H,
\end{gathered}
$$

for some $u, v^{*} \in H$. Moreover, by the estimate

$$
\varphi\left(J_{\lambda} u_{n}\right) \leq\left(\partial \varphi_{\lambda}\left(u_{n}\right), u_{n}\right) \leq \frac{1}{\lambda}\left|u_{n}\right|_{H}^{2}
$$

we see that $\varphi\left(J_{\lambda} u_{n}\right)$ is bounded. We may assume that $J_{\lambda} u_{n}$ converges to some $v$ weakly in $V$ and strongly in $H$. Then, by the demiclosedness of $\partial \varphi$, we have $\left[v, v^{*}\right] \in \partial \varphi$. Moreover, letting $n \rightarrow \infty$ in the equation $u_{n}=J_{\lambda} u_{n}+\lambda \partial \varphi_{\lambda}\left(u_{n}\right)$, we get $u=v+\lambda v^{*}$. Therefore, by the definition of $J_{\lambda}$ and $\partial \varphi_{\lambda}$, we obtain $v=J_{\lambda} u$ and $v^{*}=\partial \varphi_{\lambda}(u)$. Thus,

$$
\begin{gathered}
J_{\lambda} u_{n} \rightarrow J_{\lambda} u \quad \text { weakly in } V, \\
\partial \varphi_{\lambda}\left(u_{n}\right) \rightarrow \partial \varphi_{\lambda}(u) \quad \text { weakly in } H .
\end{gathered}
$$

Moreover, since $B$ is weakly-strongly continuous from $V$ into $H$,

$$
B\left(J_{\lambda} u_{n}\right) \longrightarrow B\left(J_{\lambda} u\right) \quad \text { strongly in } H
$$

On the other hand, by an argument similar to that of the proof of the previous lemma, we deduce from (3.23) that $t_{n} \varphi\left(u_{n}\right)$ and $t_{n}\left|B\left(u_{n}\right)\right|_{H}^{2}$ are bounded as $n \rightarrow \infty$. Hence, by (3.23), we see that $t_{n}\left|P_{i_{n}} \partial \varphi\left(u_{n}\right)\right|_{H}$ is also bounded.

If $t \neq 0$, then $\varphi\left(u_{n}\right)$ is bounded. We may assume that

$$
u_{n} \longrightarrow u \quad \text { weakly in } V \text { and strongly in } H \text {. }
$$

Therefore, it follows that $|u|_{H}=r$,

$$
\left\|T\left(u_{n}\right)\right\|_{V^{*}}
$$

is bounded,

$$
B\left(u_{n}\right) \longrightarrow B(u) \text { strongly in } H \text {. }
$$


Take a sequence $\left(w_{n}\right)$ such that $w_{n} \in H_{i_{n}}$ and $w_{n} \rightarrow u$ strongly in $V$. Then,

$$
\begin{aligned}
& t \limsup _{n \rightarrow \infty}\left\langle T\left(u_{n}\right), u_{n}-u\right\rangle_{V} \\
& \quad=\limsup _{n \rightarrow \infty}\left\langle t_{n} T_{i_{n}}\left(u_{n}\right), u_{n}-w_{n}\right\rangle_{V} \\
& \quad=-\liminf _{n \rightarrow \infty}\left(1-t_{n}\right)\left(\partial \varphi_{\lambda}\left(u_{n}\right)+B\left(J_{\lambda} u_{n}\right), u_{n}-w_{n}\right)_{H} \\
& \quad=0 .
\end{aligned}
$$

Since $T$ is of class $(S)_{+}$, it follows that $u_{n} \rightarrow u$ strongly in $V$, and hence $T\left(u_{n}\right) \rightarrow T(u)$ strongly in $V^{*}$.

We are going to show that $P_{i_{n}} T\left(u_{n}\right) \rightarrow T(u)$ weakly in $V^{*}$. Let $w$ be arbitrarily taken in $V$. Take a sequence $\left(w_{n}\right)$ such that $w_{n} \in H_{i_{n}}$ and $w_{n} \rightarrow w$ strongly in $V$. Then, noticing that $\left|P_{i_{n}} \partial \varphi\left(u_{n}\right)\right|_{H}$ is bounded, we get

$$
\begin{aligned}
\lim _{n \rightarrow \infty}\langle & \left\langle P_{i_{n}} T\left(u_{n}\right), w\right\rangle_{V} \\
& =\lim _{n \rightarrow \infty}\left(P_{i_{n}}\left(\partial \varphi\left(u_{n}\right)+B\left(u_{n}\right)\right), w-w_{n}\right)_{H}+\lim _{n \rightarrow \infty}\left(T\left(u_{n}\right), P_{i_{n}} w_{n}\right)_{H} \\
& =\lim _{n \rightarrow \infty}\left\langle T\left(u_{n}\right), w_{n}\right\rangle_{V} \\
& =\langle T(u), w\rangle_{V},
\end{aligned}
$$

which implies $P_{i_{n}} T\left(u_{n}\right) \rightarrow T(u)$ weakly in $V^{*}$. Thus, letting $n \rightarrow \infty$ in (3.23), we obtain

$$
(1-t)\left(\partial \varphi_{\lambda}(u)+B\left(J_{\lambda} u\right)\right)+t T(u)=0
$$

which contradicts (3.12) since $|u|_{H}=r$.

Now let $t=0$, that is, $t_{n} \rightarrow 0$. Then, since $t_{n}\left|B\left(u_{n}\right)\right|_{H}^{2}$ is bounded,

$$
t_{n} B\left(u_{n}\right) \longrightarrow 0 \quad \text { strongly in } H \text {. }
$$

Moreover, combining the same argument as in the proof of the previous lemma and the same one as above, we get

$$
t_{n} P_{i_{n}} \partial \varphi\left(u_{n}\right) \rightarrow 0 \quad \text { weakly in } V^{*} .
$$

Since, $t_{n}\left|P_{i_{n}} \partial \varphi\left(u_{n}\right)\right|_{H}$ is bounded, we obtain

$$
t_{n} P_{i_{n}} \partial \varphi\left(u_{n}\right) \rightarrow 0 \quad \text { weakly in } H
$$

Thus, letting $n \rightarrow \infty$ in (3.23), we obtain

$$
\partial \varphi_{\lambda}(u)+B\left(J_{\lambda} u\right)=0,
$$

which implies $u=0$.

We are going to show that $u_{n} \rightarrow u$ strongly in $H$ (and therefore $|u|_{H}=r$ ), which leads to a contradiction. 
By (3.28) and (3.35),

$$
\lim _{n \rightarrow \infty}\left(\left(1-t_{n}\right) P_{i_{n}} B\left(J_{\lambda} u_{n}\right)+t_{n} P_{i_{n}} B\left(u_{n}\right), u_{n}-u\right)_{H}=0 .
$$

Therefore, noticing that $P_{i_{n}} u \rightarrow u$ strongly in $H$, we get

$$
\begin{aligned}
\limsup _{n \rightarrow \infty} & \left(\partial \varphi_{\lambda}\left(u_{n}\right), u_{n}-u\right)_{H} \\
\quad & =\limsup _{n \rightarrow \infty}\left(\partial \varphi_{\lambda}\left(u_{n}\right), P_{i_{n}} u_{n}-P_{i_{n}} u\right)_{H}+\lim _{n \rightarrow \infty}\left(\partial \varphi_{\lambda}\left(u_{n}\right), P_{i_{n}} u-u\right)_{H} \\
& =\limsup _{n \rightarrow \infty}\left(\left(1-t_{n}\right) P_{i_{n}} \partial \varphi_{\lambda}\left(u_{n}\right), u_{n}-u\right)_{H} \\
& =-\liminf _{n \rightarrow \infty}\left(t_{n} P_{i_{n}} \partial \varphi\left(u_{n}\right), u_{n}-u\right)_{H} \\
& =-\liminf _{n \rightarrow \infty} t_{n} p \varphi\left(u_{n}\right)+\lim _{n \rightarrow \infty}\left(t_{n} P_{i_{n}} \partial \varphi\left(u_{n}\right), u\right)_{H} \\
& \leq 0 .
\end{aligned}
$$

Since $\partial \varphi_{\lambda}$ is of class $(S)_{+}$in $H$, we conclude that $u_{n} \rightarrow u$ strongly in $H$.

We have thus proved the lemma.

Let $\lambda>0$ be so small and $i \in \mathbb{N}$ be so large that (3.21) is satisfied. Then, by (2.13) and by the homotopy invariance of the Brouwer degree, we have

$$
\begin{aligned}
\operatorname{deg}_{H}\left(\partial \varphi^{p}+B_{\mu}^{p}, U_{H}(0, r)\right) & =d\left(\left(\partial \varphi_{\lambda}^{p}+B_{\mu}^{p} \circ J_{\lambda}\right)_{i}, U_{H}(0, r) \cap H_{i}\right) \\
& =d\left(\left(T_{\mu}^{p}\right)_{i}, U_{H}(0, r) \cap H_{i}\right) .
\end{aligned}
$$

Combining this equality and (3.11), we deduce (3.5).

In order to employ the homotopy invariance of $\operatorname{deg}_{H}(\cdot, \cdot)$ along $p$, we need the following lemma.

Lemma 3.4. Let $1<p_{1}<p_{2}<\infty$. Then, $\left\{\varphi^{p}: p \in\left[p_{1}, p_{2}\right]\right\}$ belongs to $\Phi_{C}^{\text {hom }}(H)$.

Proof. It is obvious that $\left\{\varphi^{p}\right\}$ satisfies (A.C) ${ }^{h}$ in Definition 2.2. We are going to show that $\left\{\varphi^{p}\right\}$ also satisfies $(\text { A.P })^{h}$.

Let $p_{n} \rightarrow p$ in $\left[p_{1}, p_{2}\right]$ and $[u, v] \in \partial \varphi^{p}$, that is,

$$
-\left(\left|u^{\prime}\right|^{p-2} u^{\prime}\right)^{\prime}=v(x), \quad x \in(0,1), u(0)=u(1)=0 .
$$

For each $n \in \mathbb{N}$, we consider the following problem:

$$
\partial \varphi^{p}\left(u_{n}\right)+\frac{1}{n}\left\{\partial \varphi^{p_{1}}\left(u_{n}\right)+\partial \varphi^{p_{2}}\left(u_{n}\right)\right\}=v .
$$

Noticing that $\partial \varphi^{q}(u)=(q-1)\left|u^{\prime}\right|^{q-2} u^{\prime \prime}$ for all $u \in D\left(\partial \varphi^{q}\right)$, we can easily see that

$$
\left(\partial \varphi^{r}(u), \partial \varphi^{q}(u)\right)_{H} \geq 0 \quad \forall u \in D\left(\partial \varphi^{r}\right) \cap D\left(\partial \varphi^{q}\right), \forall r, q \in(1, \infty) .
$$

Hence, $\partial \varphi^{p}+\left(\partial \varphi^{p_{1}}+\partial \varphi^{p_{2}}\right) / n$ becomes a maximal monotone and coercive operator in $H$, so (3.43) admits a unique solution $u_{n} \in D\left(\partial \varphi^{p}\right) \cap D\left(\partial \varphi^{p_{1}}\right) \cap D\left(\partial \varphi^{p_{2}}\right)$. Furthermore, 
since

$$
\left.|| u_{n}^{\prime}\right|^{q-2} u_{n}^{\prime \prime}|\leq|\left|u_{n}^{\prime}\right|^{p_{1}-2} u_{n}^{\prime \prime}|+|\left|u_{n}^{\prime}\right|^{p_{2}-2} u_{n}^{\prime \prime} \mid \quad \forall q \in\left[p_{1}, p_{2}\right]
$$

we find that $u_{n} \in D\left(\partial \varphi^{p_{1}}\right) \cap D\left(\partial \varphi^{p_{2}}\right) \subset D\left(\partial \varphi^{q}\right)$ for all $q \in\left[p_{1}, p_{2}\right]$, in particular, $u_{n} \in$ $D\left(\partial \varphi^{p_{n}}\right)$. Multiplying (3.43) by $u_{n}$, we get the a priori bounds for $\left|u_{n}\right|_{H}, \varphi^{p}\left(u_{n}\right), \varphi^{p_{1}}\left(u_{n}\right) / n$, and $\varphi^{p_{2}}\left(u_{n}\right) / n$. Furthermore, multiplying (3.43) by $\partial \varphi^{p}\left(u_{n}\right)$, we can deduce the a priori bound for $\left|\partial \varphi^{p}\left(u_{n}\right)\right|_{H}^{2}$. Hence, it follows from (3.43) the a priori bounds for $\left|\partial \varphi^{p_{1}}\left(u_{n}\right)\right|_{H} / n$ and $\left|\partial \varphi^{p_{2}}\left(u_{n}\right)\right|_{H} / n$. Therefore, there exists a subsequence of $\left(u_{n}\right)$ denoted again by $\left(u_{n}\right)$ such that

$$
\begin{gathered}
u_{n} \longrightarrow \hat{u} \quad \text { strongly in } H, \\
\partial \varphi_{p}\left(u_{n}\right) \rightarrow \partial \varphi_{p}(\hat{u}) \quad \text { weakly in } H, \\
\frac{\partial \varphi_{p_{1}}\left(u_{n}\right)}{n} \longrightarrow 0 \quad \text { strongly in } V_{p_{1}}^{*}, \text { and weakly in } H, \\
\frac{\partial \varphi_{p_{2}}\left(u_{n}\right)}{n} \longrightarrow 0 \quad \text { strongly in } V_{p_{2}}^{*}, \text { and weakly in } H .
\end{gathered}
$$

Hence, $\hat{u}$ should satisfy $\partial \varphi^{p}(\hat{u})=v$, which implies $\hat{u}=u$. Thus, $(\text { A.P })^{h}$ is verified with $u_{n}=u_{n}$ and $v_{n} \equiv v$.

Now, we are ready to prove Theorem 1.1.

Proof of Theorem 1.1. Let $p \in(1, \infty), \mu \in\left(\mu_{k}(p), \mu_{k+1}(p)\right)$, and $r, \rho>0$. Then, since the function $q \mapsto \mu_{k}(q)$ is continuous by $(1.2)$, there exists a continuous function $v:(1, \infty) \rightarrow \mathbb{R}$ such that

$$
\mu_{k}(q)<\nu(q)<\mu_{k+1}(q) \quad \forall q \in(1, \infty), \quad v(p)=\mu .
$$

We are going to show that

$$
\operatorname{deg}_{H}\left(\partial \varphi^{p}+B_{\nu(p)}^{p}, U_{H}(0, r)\right)=\operatorname{deg}_{H}\left(\partial \varphi^{2}+B_{\nu(2)}^{2}, U_{H}(0, r)\right)
$$

Put $p_{1}=\min \{2, p\}$ and $p_{2}=\max \{2, p\}$. Then, $\left\{B_{\mu(q)}^{q}: q \in\left[p_{1}, p_{2}\right]\right\}$ belongs to $\mathscr{B} \mathscr{D}_{1}^{\text {hom }}\left(\left\{\varphi^{q}\right.\right.$ : $\left.\left.q \in\left[p_{1}, p_{2}\right]\right\}\right)$. Indeed, the condition $(\text { A. } 2)^{h}$ in Definition 2.4 follows from the continuity of $\nu(\cdot)$. Moreover, the constant $C_{1}$ in (2.17) can be chosen independently of $q \in\left[p_{1}, p_{2}\right]$, and hence $(\mathrm{A} .3)^{h}$ is satisfied. Therefore, noticing that $\partial \varphi^{q}(u)+B_{\nu(q)}^{q}(u)=0$ has only trivial solution, we deduce (3.48) from the homotopy invariance of $\operatorname{deg}_{H}(\cdot, \cdot)$. By (3.5) and (3.48), we get

$$
\begin{aligned}
\operatorname{deg}_{(S)_{+}}\left(T_{\mu}^{p}, U_{V_{p}}(0, \rho)\right) & =\operatorname{deg}_{H}\left(\partial \varphi^{p}+B_{v(p)}^{p}, U_{H}(0, r)\right) \\
& =\operatorname{deg}_{H}\left(\partial \varphi^{2}+B_{\nu(2)}^{2}, U_{H}(0, r)\right) \\
& =\operatorname{deg}_{(S)_{+}}\left(T_{\nu(2)}^{2}, U_{V_{2}}(0, \rho)\right) .
\end{aligned}
$$

It is shown in the proof of [6, Proposition 4.2] that the last equation is equal to $(-1)^{k}$.

We have thus completed the proof of Theorem 1.1. 
Corollary 3.5. Let $\mu \in\left(\mu_{k}(p), \mu_{k+1}(p)\right)$ for some $k \in \mathbb{N} \cup\{0\}$. Then,

$$
\operatorname{deg}_{H}\left(\partial \varphi^{p}+B_{\mu}^{p}, U_{H}(0, r)\right)=(-1)^{k} \quad \forall r>0, \forall p \in(1, \infty) .
$$

\section{Application}

Consider the following variational inequality:

Find

$$
\begin{aligned}
u \in K: \int_{0}^{1}\left|u^{\prime}(x)\right|{ }^{p-2} u^{\prime}(x)\left(v^{\prime}(x)-u^{\prime}(x)\right) d x & \\
& \geq \int_{0}^{1} f(x, u(x))(v(x)-u(x)) d x \quad \forall v \in K,
\end{aligned}
$$

where $1<p<\infty, f:(0,1) \times \mathbb{R} \rightarrow \mathbb{R}$ is a Carathéodory function and

$$
K=\left\{v \in W_{0}^{1, p}(0,1):\left|v^{\prime}(x)\right| \leq C \text { a.e. in } \Omega\right\}
$$

for some $C>0$. In [11], the higher-dimensional version of (4.1) is considered, where the multiple existence of solutions is shown under the condition that $\lim _{\tau \rightarrow 0} f(x, \tau) /|\tau|^{p-2} \tau$ lies between the first and the second eigenvalues of $p$-Laplacian in a sense.

As for the one-dimensional case, we have the following theorem.

THEOREM 4.1. Suppose that $f$ satisfies the following conditions:

(i) for each $r>0$, there exists a function $\rho_{r} \in L^{1}(\Omega)$ such that

$$
|f(x, \tau)| \leq \rho_{r}(x) \quad \text { a.e. } x \in(0,1), \forall \tau \in \mathbb{R} \text { with }|\tau| \leq r
$$

(ii) it holds that for some $k \geq 1$,

$$
\mu_{k}(p) \leq \equiv \lim _{\tau \rightarrow 0} \frac{f(x, \tau)}{|\tau|^{p-2} \tau} \leq \not \equiv \mu_{k+1}(p) \quad \text { a.e. } x \in(0,1) .
$$

Then, the variational inequality (4.1) has at least three solutions.

Proof. Since a proof is quite similar to that of [11, Theorem 4.1], we only give an outline of it. We employ the degree for $(\mathrm{S})_{+}$-mappings with maximal monotone perturbations. It will be also denoted by $\operatorname{deg}_{(S)_{+}}(\cdot, \cdot)$.

Since $K$ forms a bounded subset of $L^{\infty}(0,1)$, we may assume that

$$
|f(x, \tau)| \leq \rho(x) \text { a.e. } x \in(0,1), \forall \tau \in \mathbb{R},
$$

for some $\rho \in L^{1}(0,1)$ by cutting off the function $f$ with respect to the second variable.

Set $V=W_{0}^{1, p}(0,1)$, and define the mapping $S_{f}^{p}: V \rightarrow V^{*}$ by

$$
\left\langle S_{f}^{p}(u), v\right\rangle_{V}=\int_{0}^{1}\left|u^{\prime}\right|^{p-2} u^{\prime} v^{\prime} d x-\int_{0}^{1} f(x, u) v d x \quad u, v \in V .
$$


Then, $S_{f}^{p}$ becomes of class $(S)_{+}$since, by $(4.5)$, the mapping $u \mapsto f(\cdot, u)$ is compact from $V$ into $V^{*}$. Hence, the variational inequality $(4.1)$ can be reduced to the following abstract equation in $V^{*}$ :

$$
S_{f}^{p}(u)+\partial_{V} I_{K}(u) \ni 0,
$$

where $I_{K}$ is the indicator function of $K$ and $\partial_{V} I_{K}: V \rightarrow V^{*}$ is its subdifferential in $V$. Moreover, $u$ is a solution of (4.7) if and only if it is a critical point of the functional $\Psi+I_{K}$, where

$$
\Psi(u)=\frac{1}{p} \int_{0}^{1}\left|u^{\prime}\right|^{p} d x-\int_{0}^{1} \int_{0}^{u(x)} f(x, \tau) d \tau d x, \quad u \in V .
$$

We proceed to find three solutions of (4.7). The first solution is $u=0$ since $f(x, 0)=0$ by (4.4). The second one is the global minimizer of $\Psi+I_{K}$. Indeed, $\Psi+I_{K}$ is bounded below by (4.5), and its infimum is achieved by some $u_{0} \in K$ since $K$ is bounded and $\Psi+I_{K}$ is weakly lower semicontinuous. Moreover, it easily follows from $(4.4)$ that $\Psi\left(u_{0}\right)<0=$ $\Psi(0)$, which implies $u_{0} \neq 0$.

We may assume that $u_{0}$ is an isolated solution for (4.7), otherwise the assertion of the theorem holds. Therefore, by [11, Theorem 3.13], we get

$$
\operatorname{deg}_{\left(S_{+}\right.}\left(S_{f}^{p}+\partial_{V} I_{K}, U_{V}\left(u_{0}, r\right)\right)=1
$$

for small $r>0$. Noticing the fact that $K$ is bounded, we also have

$$
\operatorname{deg}_{(S)_{+}}\left(S_{f}^{p}+\partial_{V} I_{K}, U_{V}(0, R)\right)=1
$$

for large $R>0$. Moreover, from (4.4), we can derive

$$
\operatorname{deg}_{(S)_{+}}\left(S_{f}^{p}+\partial_{V} I_{K}, U_{V}(0, r)\right)=\operatorname{deg}_{(S)_{+}}\left(T_{\mu}^{p}, U_{V}(0, r)\right)
$$

for small $r>0$ and for any $\mu \in\left(\mu_{k}(p), \mu_{k+1}(p)\right)$ (use [3, Proposition 5.1] instead of [11, Lemma 4.2]). Therefore, by Theorem 1.1, we get

$$
\operatorname{deg}_{(S)_{+}}\left(S_{f}^{p}+\partial_{V} I_{K}, U_{V}(0, r)\right)=(-1)^{k}
$$

for small $r>0$. Finally, by (4.9), (4.10), and (4.12), we have

$$
\begin{aligned}
\operatorname{deg}_{\left(S_{+}\right)_{+}} & \left(S_{f}^{p}+\partial_{V} I_{K}, U_{V}(0, R) \backslash \overline{U_{V}\left(u_{0}, r\right) \cup U_{V}(0, r)}\right) \\
= & 1-1-(-1)^{k} \neq 0
\end{aligned}
$$

which assures the existence of the third solution. 


\section{Acknowledgments}

The first author was supported by Waseda University Grant for Special Research Projects, no.2003A-086. The second author was partially supported by the Grants-in-Aid for Scientific Research, no.12440051 and no.15654024, the Ministry of Education, Culture, Sports, and Technology, Japan, and by Waseda University Grant for Special Research Projects, no.2003B-027.

\section{References}

[1] H. Brézis, Opérateurs Maximaux Monotones et Semi-Groupes de Contractions dans les Espaces de Hilbert, North-Holland Mathematics Studies, vol. 5, North-Holland Publishing, Amsterdam, 1973.

[2] F. E. Browder, Fixed point theory and nonlinear problems, Bull. Amer. Math. Soc. (N.S.) 9 (1983), no. $1,1-39$.

[3] M. del Pino, M. Elgueta, and R. Manásevich, A homotopic deformation along $p$ of a LeraySchauder degree result and existence for $\left(\left|u^{\prime}\right|^{p-2} u^{\prime}\right)^{\prime}+f(t, u)=0, u(0)=u(T)=0, p>1$, J. Differential Equations 80 (1989), no. 1, 1-13.

[4] M. del Pino and R. Manásevich, Global bifurcation from the eigenvalues of the p-Laplacian, J. Differential Equations 92 (1991), no. 2, 226-251.

[5] P. Drábek and N. M. Stavrakakis, Bifurcation from the first eigenvalue of some nonlinear elliptic operators in Banach spaces, Nonlinear Anal. Ser. A: Theory Methods 42 (2000), no. 4, 561572.

[6] N. Hirano, Multiple solutions for quasilinear elliptic equations, Nonlinear Anal. 15 (1990), no. 7, 625-638.

[7] A. G. Kartsatos and I. V. Skrypnik, The index of a critical point for nonlinear elliptic operators with strong coefficient growth, J. Math. Soc. Japan 52 (2000), no. 1, 109-137.

[8] The index of a critical point for densely defined operators of type $\left(S_{+}\right)_{L}$ in Banach spaces, Trans. Amer. Math. Soc. 354 (2002), no. 4, 1601-1630.

[9] A. G. Kartsatos, I. V. Skrypnik, and V. N. Shramenko, The index of an isolated critical point for a class of non-differentiable elliptic operators in reflexive Banach spaces, to appear in J. Differential Equations.

[10] J. Kobayashi and M. Ôtani, Degree for subdifferential operators in Hilbert spaces, Adv. Math. Sci. Appl. 14 (2004), no. 1, 307-325.

[11] applications to variational inequalities, Nonlinear Anal. 59 (2004), no. 1-2, 147-172.

[12] M. Ôtani, A remark on certain nonlinear elliptic equations, Proc. Fac. Sci. Tokai Univ. 19 (1984), $23-28$.

[13] I. V. Skrypnik, Methods for Analysis of Nonlinear Elliptic Boundary Value Problems, Translations of Mathematical Monographs, vol. 139, American Mathematical Society, Rhode Island, 1994, translated from the 1990 Russian original by D. D. Pascali.

Jun Kobayashi: Department of Applied Physics, School of Science and Engineering, Waseda University, 3-4-1 Okubo, Shinjuku-ku, Tokyo 169-8555, Japan

E-mail address: kobajun_chanta@yahoo.co.jp

Mitsuharu Ôtani: Department of Applied Physics, School of Science and Engineering, Waseda University, 3-4-1 Okubo, Shinjuku-ku, Tokyo 169-8555, Japan

E-mail address: otani@waseda.jp 


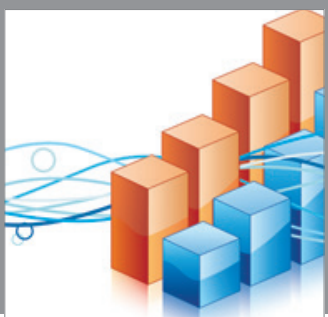

Advances in

Operations Research

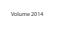

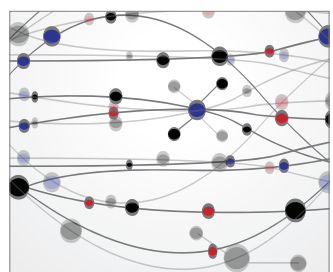

\section{The Scientific} World Journal
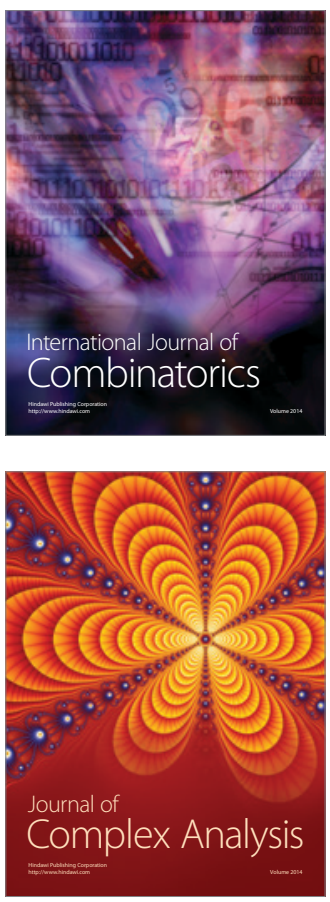

International Journal of

Mathematics and

Mathematical

Sciences
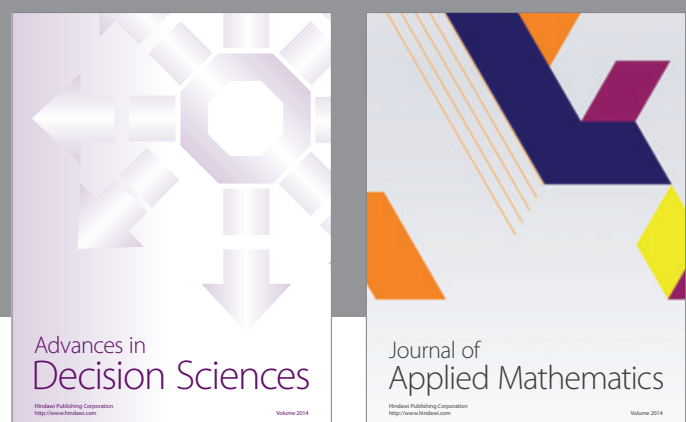

Journal of

Applied Mathematics
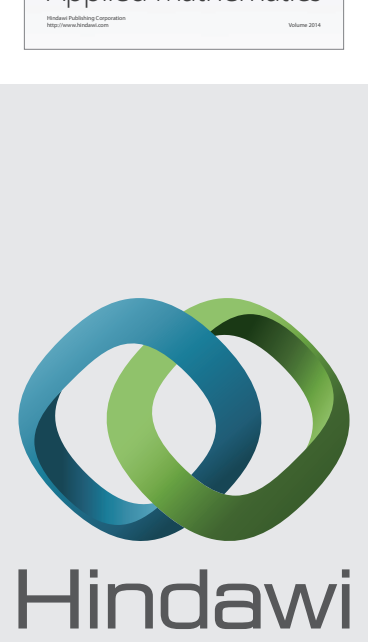

Submit your manuscripts at http://www.hindawi.com
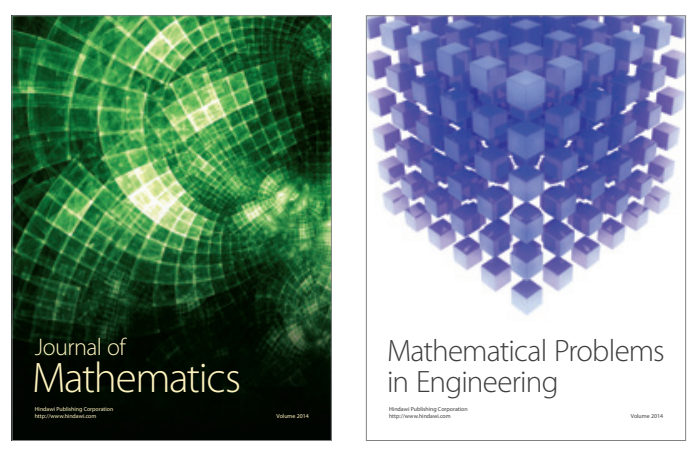

Mathematical Problems in Engineering
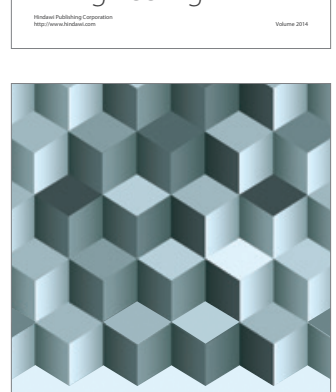

Journal of

Function Spaces
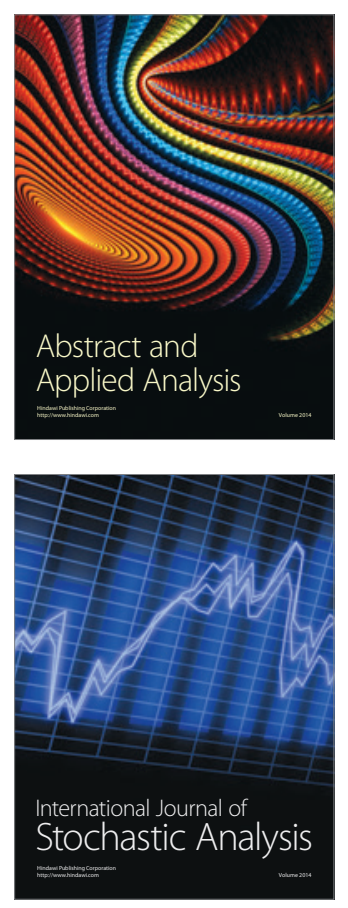

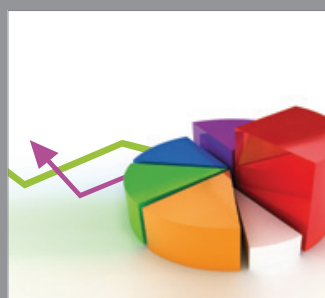

ournal of

Probability and Statistics

Promensencen
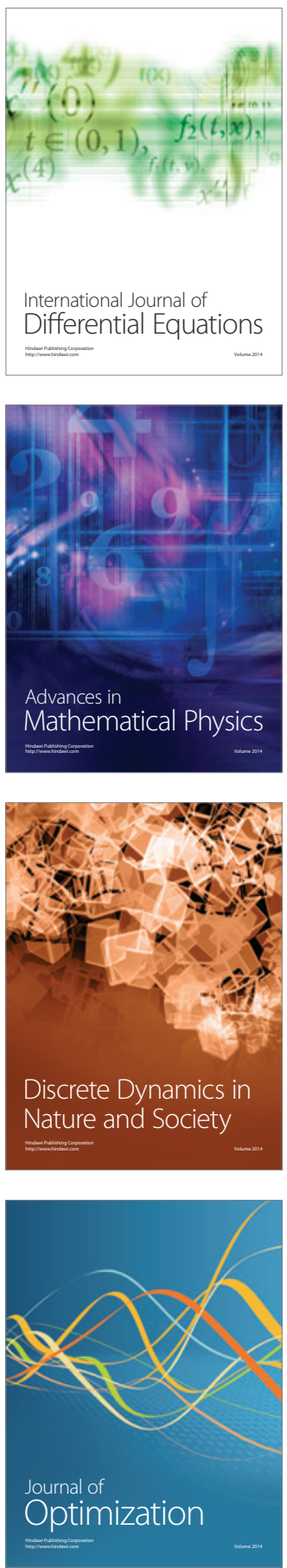